\title{
Malnutrition in Developing Countries, From Undernutrition to Obesity!
}

\author{
Amar Al Shibli ${ }^{1}$ and Haydar Jawad Al Rufaye ${ }^{2 *}$ \\ ${ }^{1}$ Consultant Pediatrician, Tawam Hospital, UAE \\ ${ }^{2}$ Pediatric Resident, Tawam Hospital, UAE
}

*Corresponding author: Haydar Jawad Al Rufaye, Consultant Pediatrician,

Tawam Hospital, UAE.

Received Date: August 08, 2019

Published Date: August 21, 2019

\section{Opinion}

Malnutrition is defined as deficiencies, excesses, or imbalances in a person's intake of energy and/or nutrients. It addresses 3 conditions which are; undernutrition, micronutrient-related malnutrition and overweight (WHO 2019). Every country in the world has one or more forms of malnutrition and combating malnutrition has always been one of the most important goals of healthcare.

Classically, undernutrition has been the biggest culprit in malnutrition. Wasting and Stunting have been widely used as measures for undernutrition. Low weight-for-height is known as wasting, it usually indicates recent and severe weight loss. On the other hand, low height-for-age is known as stunting and it is the result of chronic or recurrent undernutrition, usually associated with poor socioeconomic conditions, poor maternal health and nutrition. Children with low weight-for-age are known as underweight. A child who is underweight may be stunted, wasted, or both.

Underweight is a very common problem, in 2018, $21.9 \%$ of children under age 5 worldwide had stunted growth. The good news is that this number has declines significantly from the year 2000. Between 2000 and 2018, stunting prevalence globally has declined from $32.5 \%$ to $21.9 \%$, and the number of children affected fell from 198.2 million to 149.0 million. Most of the underweight children live in the developing countries, in 2018, nearly two out of five stunted children lived in South Asia while another two out of five lived in sub-Saharan Africa.

Underweight puts the child at serious risk of infection, this can lead to cycle of worsening illness and deteriorating nutritional status. Poor nutrition can also lead to stunted growth, impaired cognitive ability and reduced school and work performance.
Multiple efforts have been put to fight and stop undernutrition worldwide, these efforts have been a large success as evident by the numbers provided earlier, but the road is still long. However, now, a new trend is rising. Obesity and overweight throughout the world are on the rise.

In $2018,5.9 \%$ of children worldwide or 40.1 million children were overweight or obese, this number is an increase from $4.9 \%$ or 30.1 million child in 2000. Surprisingly this number is worldwide, and it includes regions of undeveloped countries, for example, South Asia which already has a very high prevalence of undernutrition, as stated earlier, has seen a rise in overweight children where the prevalence has increased from $2.5 \%$ to $3.1 \%$.

Overweight and obesity result from an increase in energy consumption and associated in sedentary lifestyle. It is widely established that obesity puts children at risk of cardiovascular diseases, malignancy and diabetes. Unhealthy diets and poor nutrition are among the top risk factors for these diseases globally.

In the developed countries, there are many ongoing researches on the prevalence, risks and complications of diet related diseases. Extensive work on all levels was done to prevent the obesity incidence from increase. However; the incidence of obesity is still increasing. In the developing countries, there are no much data available to assess the prevalence of malnutrition; however, we are noticing increasing in the obesity side especially in the rich counties, on the other hand the prevalence of undernutrition is till high and may probably see an increase later on in the future because of shift from undernutrition to obesity.

An extensive work needs to be done to try to eliminate malnutrition, we also need to make sure and prevent shifting undernutrition to overweight side. 
In conclusion, malnutrition continues to be a leading cause of morbidity and mortality. Undernutrition is the most prevalent form of malnutrition still, but the prevalence is decreasing. On the other hand, overweight and obesity is increasing even in the underdeveloped countries. This major shift will require further researches and efforts especially in the developing countries.

\section{Acknowledgement}

None.

\section{Conflict of Interest}

No conflict of interest. 